\title{
AN INEQUALITY ABOUT PAIRS OF CONJUGATE HÖLDER NUMBERS
}

\author{
AndRes FERnANDEZ AND Aurel I. StAn
}

Abstract. Most of the inequalities that we encounter in mathematics are based on a monotonicity or convexity argument. The functions that are constructed during a proof are monotone or convex (concave) throughout their domains. However, there are functions that change their monotonicity and convexity on their domains. In this case, a chopping of the domain into intervals on which a function is monotone or convex (concave) is necessary. Two inequalities about pairs of Hölder conjugate numbers are presented. One follows very elegantly from Young inequality, and the other requires chopping the domain into three subintervals, and proving the inequality differently on each of them.

Mathematics subject classification (2010): 26 D15.

Keywords and phrases: Young inequality, Bernoulli inequality, Taylor formula with Lagrange remainder, Jensen inequality, monotone and convex functions.

\section{REFERENCES}

[1] F. BARTHE, Optimal Young's Inequality and its convers: a simple proof, Geom. funct. anal., 8, (1998), 234-242.

[2] W. BECKNER, Inequalities in Fourier Analysis, Ann. of Math., 102, (1975), 159-182.

[3] H. J. BRASCAMP AND E. H. LIEB, Best constants in Young's inequality, its converse, and its generalization to more than three functions, Adv. in Math. 20, (1976), 151-173.

[4] E. H. LiEB, Gaussian kernels have only Gaussian maximizers, Invent. Math., 102, (1990), 179-208.

[5] E. H. Lieb And M. Loss Analysis, Graduate Studies in Mathematics, 14, Second Edition, AMS, Providence, Rhode Island, 2001. 